\title{
EFICIÊNCIA DE UM FILTRO DE MANGAS NO TRATAMENTO DE GASES ORIUNDOS DE CALDEIRA DE QUEIMA DE BIOMASSA SÓLIDA
}

\author{
Marcelo Garcia Barbosa ${ }^{1}$ \\ Celso Luiz da Silva ${ }^{2}$
}

RESUMO: Atualmente a implantação de qualquer planta industrial que possa gerar impacto ambiental, ou até mesmo adequação de equipamentos já instalados para a não geração de poluentes ou geração dentro de limites adequados, passa cada vez mais por fiscalização rigorosa dos órgãos ambientais. A ventilação industrial tem sido e continua sendo a principal medida de controle efetiva para ambientes de trabalho que contenham dispersão de efluentes gasosos e material particulado de natureza prejudiciais, e baseado neste fato de relevância inegável é que se fundamenta este estudo. Um sistema de tratamento de gases com enfoque em ventilação local exaustora engloba vários equipamentos, e dentre eles o filtro de mangas é considerado um dos principais, pois sua função é a de efetuar a filtração dos gases proporcionando emissões dentro das tolerâncias dos órgãos ambientais, refletindo desta forma o objeto principal deste estudo de caso, a eficiência do equipamento. Especificamente neste estudo apresentam-se dados de eficiência do filtro de mangas em questão, componente de um sistema de desempoeiramento de uma caldeira de queima de resíduos sólidos. Foram utilizados dados coletados em campo em dois pontos do circuito do sistema, entrada do filtro de mangas e descarga de gases limpos, além de análise do elemento filtrante. As coletas e análises foram realizadas de acordo com as normas para a correta aferição dos dados gerados. Os resultados permitiram observar patamares de eficiência de filtração para os resíduos gerados na queima, demonstrando os cuidados a serem tomados no dimensionamento do equipamento para esta aplicação.

Palavras- chave: filtro de mangas. Tratamento de gases. Eficiência.

\footnotetext{
${ }^{1}$ Engenheiro Mecânico, Unesp - Universidade Estadual Paulista - Câmpus de Bauru. marcelo_gb@yahoo.com.br.

${ }^{2}$ Doutor, Unesp - Universidade Estadual Paulista - Câmpus de Bauru. celsos@feb.unesp.br.
} 


\section{INTRODUÇÃO}

Com o advento de normas e restrições cada vez mais rígidas por parte dos órgãos ambientais, em virtude da crescente preocupação com as modificações climáticas recentes geradas pela contínua descarga de emissões poluentes na atmosfera, torna-se essencial a implantação de equipamentos que amortizem ou eliminem esses resíduos. Para este estudo, foi implantado um sistema de tratamento de gases pós-caldeira de queima de resíduos sólidos (bagaço de cana), onde durante o período de testes foi observado uma série de fatores e dados que serão explanados mais adiante. O principal objetivo deste experimento foi verificar a possibilidade de implantação de um sistema de desempoeiramento a seco para caldeira de queima de biomassa, visto que para esta aplicação específica um sistema de desempoeiramento úmido é o mais usual. Ao se verificar esta possibilidade visava-se obter vantagens econômicas e técnicas, tais como:

- redução de custos,

- atender futuras exigências dos órgãos ambientais com tendência a serem cada vez mais restritas,

- aumento de eficiência de filtragem de particulados,

- eliminação de efluentes secundários.

Tendo a eficiência de filtragem como tema deste trabalho, o principal componente deste experimento torna-se então o filtro de mangas, agente responsável pela alta eficiência de filtragem alcançada em sistemas a seco de filtração. Os dados do filtro utilizado estão dispostos a seguir na tabela 1.

Tabela 1. Dados do filtro de mangas Dinaflux 8x7-30

\begin{tabular}{c|c}
\hline Modelo & Dinaflux $8 \times 7-30$ \\
\hline Vazão & $5.000 \mathrm{~m}^{3} / \mathrm{h}$ \\
Temperatura & $230^{\circ} \mathrm{C}$ \\
Dimensional das Mangas & $160 \times 3.000 \mathrm{~mm}$ \\
Número de Mangas & 56 \\
Velocidade de Filtragem & $58,4 \mathrm{~m}^{3} / \mathrm{m}^{2} . \mathrm{h}$ \\
Área Filtrante & $85,61 \mathrm{~m}^{2}$ \\
Velocidade de Ascensão & $0,54 \mathrm{~m} / \mathrm{s}$ \\
Tecido das Mangas & Goretex (W. L. GORE) \\
& Pecido de fibra de vidro com membrana expandida de \\
& PTFE (Teflon) \\
\hline
\end{tabular}


A implantação do projeto piloto em questão foi realizada desviando-se somente parte do fluxo dos gases da caldeira, tendo em vista o caráter experimental do sistema de tratamento de gases. Este desvio de fluxo foi implementado instalando-se um duto adjacente ao duto de saída de gases do processo.

$\mathrm{Na}$ figura 1 apresenta-se a imagem da instalação piloto, com a respectiva disposição dos equipamentos utilizados em uma montagem típica para sistemas de tratamento de gases por via seca.

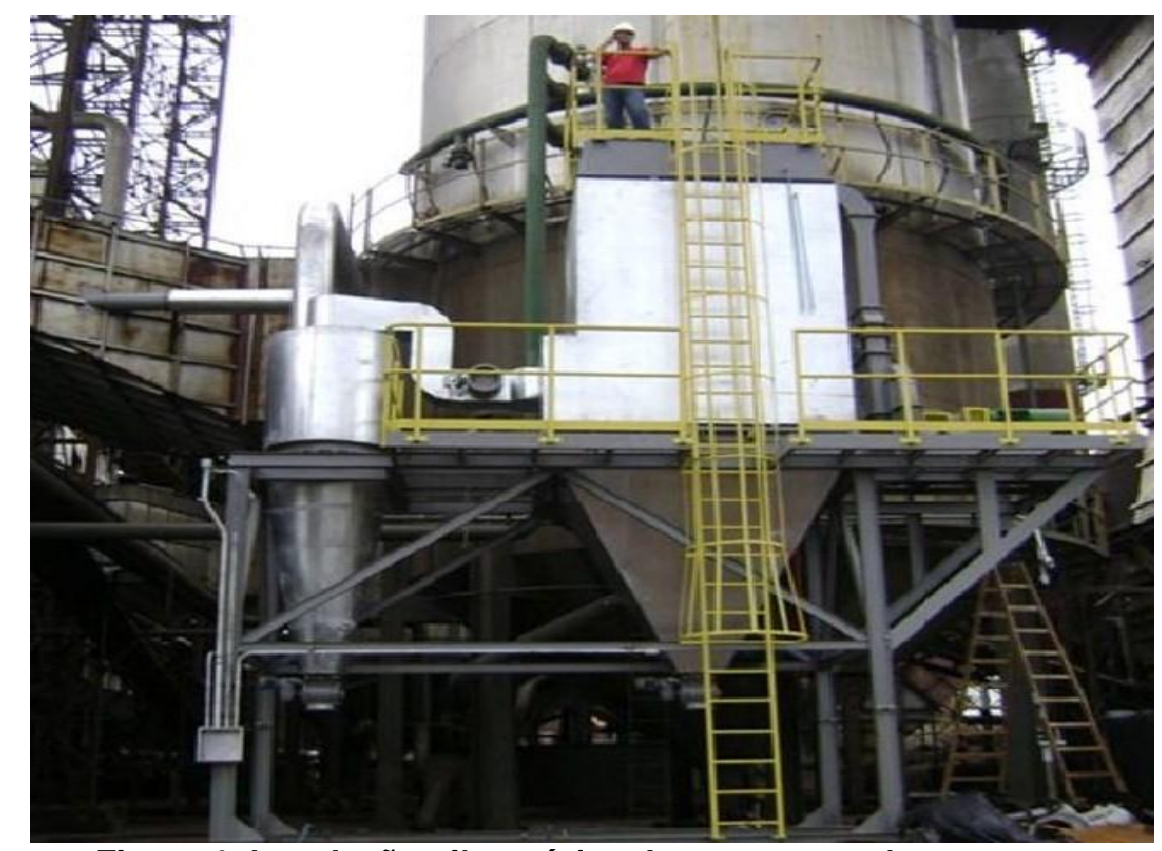

Figura 1. Instalação piloto típica de tratamento de gases

\section{MATERIAIS E MÉTODOS}

Adotou-se para este experimento uma típica instalação de sistema de desempoeiramento e tratamento de gases via seca como relatado. Os gases advindos do processo (caldeira de queima de biomassa) são transportados através de duto em aço carbono, que interliga a caldeira ao precipitador do tipo ciclone instalado anteriormente ao coletor do tipo filtro de mangas. No duto de interligação entre o precipitador e o coletor 
utilizou-se uma válvula controladora de fluxo do tipo damper borboleta, com acionamento por atuador eletrônico motorizado com posicionador, equipamento responsável pela proteção do filtro de mangas em caso de excessiva temperatura dos gases, atuando de forma a permitir a entrada de ar ambiente $\left(30^{\circ} \mathrm{C}\right)$ no circuito do duto de transporte de gases, o que resulta em uma mistura com os gases do processo de temperatura inferior a dos gases provenientes da caldeira. Conduzidos até o filtro de mangas, que L"offler, F.; Dietrich, H.W. (1988), mostra ser utilizado para remoção de particulado sólido dos gases do processo, os gases são filtrados e com quantidade de poluentes reduzidos são encaminhados através de dutos pelo uso de ventilador centrífugo para a chaminé do sistema. O filtro de mangas utilizado é do tipo jato pulsante "on-line" que trabalha com pressão negativa. A figura 2 ilustra um fluxograma do sistema descrito.

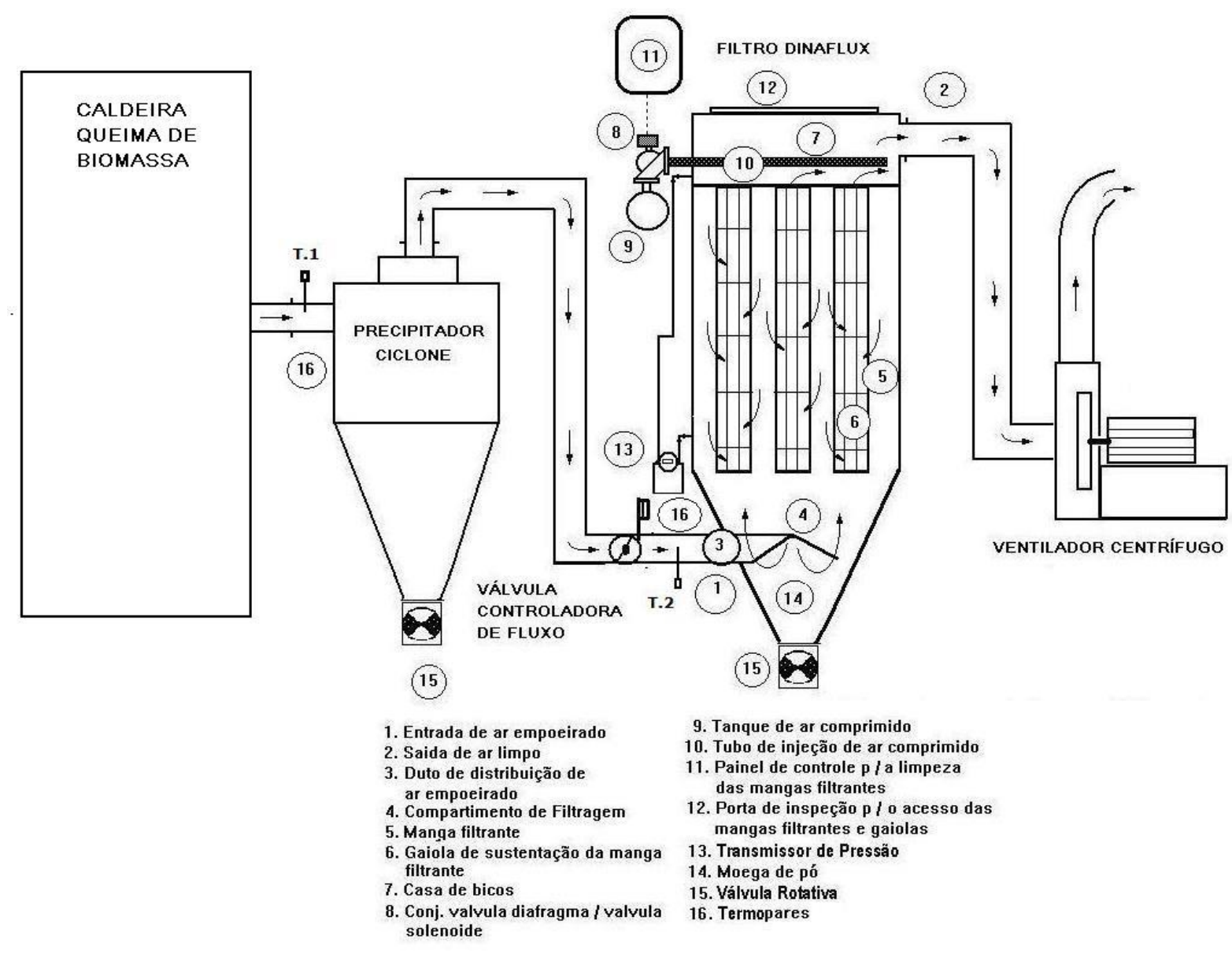

Figura 2. Fluxograma do sistema de tratamento de gases 
Todo o sistema foi monitorado por instrumentação, que inclui sensores termopar T.1 e T.2 (item 16 da figura 2) e filtro de mangas que contava com transmissor de pressão diferencial. O controle do sistema foi efetuado via controlador lógico programável (C.L.P.), onde foi realizada a coleta de dados para os parâmetros citados durante todo o período de funcionamento.

O sistema de tratamento de gases foi operado todo o tempo de forma usual para sistemas a seco, sendo seus parâmetros base de funcionamento inseridos no C.L.P. O sistema de limpeza jato pulsante, alimentado por linha de ar comprimido com pressão de 6 bar, que American Conference of Governmental Industrial Hygienists - Committee on Industrial Ventilation (2001) mostra ser valor usual para este tipo de sistema de limpeza (5,52 bar a 6,89 bar), era iniciado levando-se em conta o aumento excessivo da queda de pressão através do filtro de mangas. O isolamento térmico dos dutos de transporte de gases para evitar queda de temperatura do fluxo e possível condensação foi realizado. $O$ advento do precipitador instalado anteriormente ao coletor deu-se devido à necessidade de proteção dos elementos filtrantes contra eventuais fagulhas incandescentes provenientes da caldeira, pois uma única fagulha junto ao material coletado acumulado é suficiente para início de combustão do material. A operação do sistema de tratamento de gases iniciou-se em maio de 2008, sendo que todos os percalços de ajustes do equipamento foram sanados antes do período efetivo de testes, e em maio de 2010 o sistema passou a trabalhar em regime contínuo. Após aproximadamente dois meses em funcionamento, período utilizado para alcançar o nível de saturação suficiente dos elementos filtrantes de acordo com Mesquita, A.L.S.; Guimarães, F.A.; Nefussi, N. (1977), que mostra que para filtros de tecidos sem utilização (eficiência de coleta da ordem de $60 \%$ ) a eficiência de coleta aumenta para valores acima de 90\% tão logo material particulado seja depositado, os dados de pressão e temperatura passaram a ser coletados considerando a situação ideal de filtração.

De forma a complementar os dados de pressão e temperatura coletados, a retirada de elemento filtrante para análise foi realizada ao final do período de testes (cinco meses de operação). Na tabela 2 podem ser encontradas maiores informações a respeito dos 
métodos utilizados na análise do elemento filtrante, cujo próprio fabricante foi o agente realizador dos testes.

Tabela 2. Método de análise do elemento filtrante (manga)

\begin{tabular}{ll}
\hline Método de Coleta da Manga Filtrante & -Coleta aleatória no Filtro Dinaflux 8x7-30 \\
\hline & -Tecido de fibra de vidro com gramatura de $750 \mathrm{~g} / \mathrm{m}^{2} \mathrm{e}$ \\
Características da Manga & membrana expandida de PTFE com fixação por anel de \\
& aço mola \\
& -Gramatura \\
Análises Realizadas & -Permeabilidade \\
& -Resistência a tração \\
& -Alongamento \\
& -Análise visual \\
\hline
\end{tabular}

Também foram efetuadas medições de efluentes no duto de entrada do filtro de mangas e na descarga de ar limpo deste ao final do período de testes. A coleta dos gases utilizados para análise dos efluentes foi feita com sistema para amostragens isocinéticas, tendo o gasômetro seco, placa de orifício e tubo de pitot calibrados, e foram realizadas de forma simultânea na entrada e saída do filtro de mangas para que pudessem gerar comparações plausíveis com os dados obtidos. As análises assim como as coletas foram realizadas por empresa especializada e credenciada. Na tabela 3 encontra-se maiores informações sobre a coleta realizada.

Tabela 3. Método de medições de efluentes

\begin{tabular}{ll}
\hline Local de Coleta & -Duto de Entrada do Filtro de Mangas (Gases com \\
& $\begin{array}{l}\text { Particulado) } \\
\text {-Duto de Descarga do Filtro de Mangas (Gases Limpos) }\end{array}$ \\
\hline \multirow{2}{*}{ Análises Realizadas } & -Emissão de Material Particulado \\
& -Emissão de Óxidos de Nitrogênio \\
& -Material Particulado: pesagem do material retido no \\
& filtro, sonda e ciclone \\
& -Óxidos de Nitrogênio: método colorimétrico, utilizando \\
& espectrofotômetro UV \\
& -Gases de Emissão: coletas efetuadas nos sacos de tedlar. \\
& Os teores de Oxigênio, Dióxido de Carbono e Nitrogênio \\
nos gases foram obtidos por meio de dosagem & \\
& volumétrica pela técnica de Orsat.
\end{tabular}




\section{RESULTADOS E DISCUSSÃO}

Tanto a análise dos gases quanto do elemento filtrante, juntamente ao acompanhamento dos dados de temperatura e pressão realizado junto ao C.L.P. do sistema de tratamento de gases, permite que se chegue a uma série de conclusões e ponderações sobre a performance alcançada pelo filtro de mangas. Por ter a função de ser o coletor principal do sistema, os cuidados e observações a serem efetuadas no dimensionamento do filtro de mangas abrangem variados aspectos. Considerações a respeito de carga de pó, temperatura, umidade, velocidade de ascensão dos gases na câmara suja e velocidade de filtragem dos gases devem ser corretamente ponderadas. Um dos principais pontos a serem observados é a determinação da velocidade de filtragem adequada, que Saleem, M.; Krammera, G. (2007) mostra ter grande influência na queda de pressão através do equipamento e formação da camada de pó externa ao elemento filtrante (cake).

$\mathrm{O}$ enfoque evidenciado neste trabalho sobre o coletor justifica-se pelo fato de 0 processo gerador dos gases ser comumente desempoeirado via úmida, possuindo a composição dos gases oriundos da caldeira alta umidade e altíssima geração de fagulhas incandescentes, fatores extremamente prejudiciais em filtrações via seca com elementos filtrantes em tecido. Um dos principais fatores para que se possa atestar a possibilidade de utilização deste tipo de equipamento no tratamento de gases deste processo, são os valores de queda de pressão alcançados durante a operação em regime normal contínuo. Os valores de temperatura e pressão coletados durante o período de testes no sistema estão na figura 3, onde T1 é a temperatura na captação dos gases, T2 é a temperatura na entrada do filtro e $\Delta \mathrm{P}$ é a queda de pressão através do equipamento (valores médios do dia de coleta). 

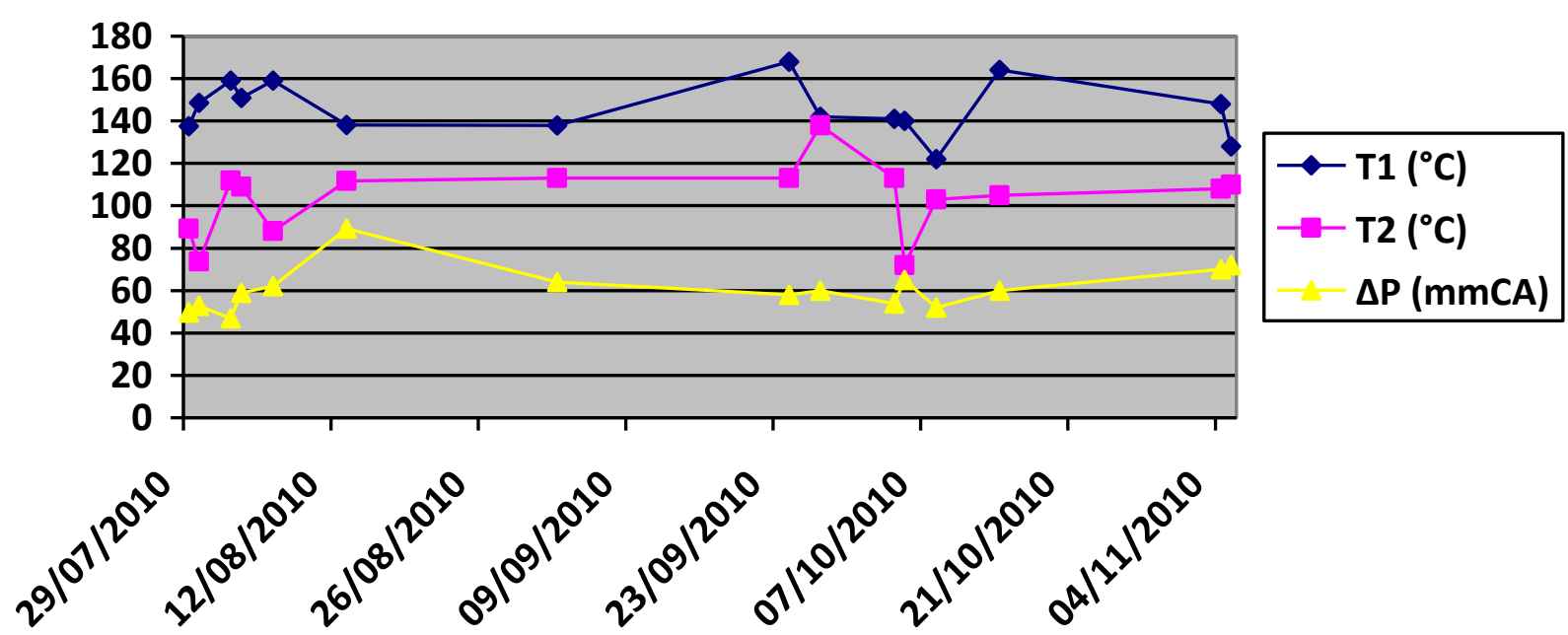

Figura 3. Dados coletados de pressão e temperatura ao longo do experimento

Observa-se na figura 3 que os valores de temperatura monitorados não apresentaram picos suficientes para que houvesse o acionamento da válvula de controle de fluxo para entrada de ar de diluição (ar ambiente) no fluxo de gases do circuito, visto que a temperatura máxima de projeto permitida no filtro de mangas era de $230^{\circ} \mathrm{C}$. Os valores de pressão monitorados foram mantidos, de acordo com a figura 3, sempre abaixo de $90 \mathrm{mmCA}$, demonstrando uma das características principais dos coletores desta categoria, em acordo com alguns trabalhos (Mesquita, A.L.S.; Guimarães, F.A.; Nefussi, N, 1977; American Conference of Governmental Industrial Hygienists - Committee on Industrial Ventilation 2001,) que mostram ser uma queda de pressão não excessiva no equipamento esta característica. Estes valores de queda de pressão viabilizam então a possibilidade de uma instalação com menor potência instalada, pois a perda de carga final do circuito (dutos, ciclone, filtro de mangas, duto de descarga) a ser suprida pelo ventilador centrífugo será também menor. Pode-se concluir então diante destes dados de queda de pressão coletados, que os valores de velocidade de ascensão dos gases na câmara suja e velocidade de filtragem considerados são então adequados para a aplicação em estudo, pois geraram em conjunto condições suficientes para o correto funcionamento do equipamento em detrimento logicamente da carga de pó a ser filtrada. 
Visto e analisado o funcionamento do filtro de mangas por este âmbito, é conveniente e necessário que se verifique a eficiência alcançada, fator preponderante para o objetivo final de um equipamento de tratamento de gases, tendo como base 0 correto funcionamento demonstrado. Para filtros de tecidos, que American Conference of Governmental Industrial Hygienists - Committee on Industrial Ventilation , (2001), mostra ter eficiência de mais de 99\% para material particulado, uma das principais características é justamente os valores baixíssimos de emissão alcançados na saída do equipamento diante das crescentes requisições dos órgãos ambientais. Nas tabelas 4 e 5 estão dispostos os valores encontrados na coleta na entrada do filtro de mangas.

Tabela 4. Emissão de material particulado na entrada do filtro de mangas

\begin{tabular}{|c|c|c|c|}
\hline Parâmetro & $\begin{array}{c}\text { Amostragem } \\
1\end{array}$ & $\begin{array}{c}\text { Amostragem } \\
2\end{array}$ & Média \\
\hline Concentração Condições Normais Base Seca $\left(\mathrm{mg} / \mathrm{Nm}^{3}\right)$ & 6.781 & 2.242 & 4.511 \\
\hline Concentração Corrigida a $7 \% \mathrm{O}_{2}\left(\mathrm{mg} / \mathrm{Nm}^{3}\right)$ & 10.432 & 3.375 & 6.904 \\
\hline Concentração Corrigida a $8 \% \mathrm{O}_{2}\left(\mathrm{mg} / \mathrm{Nm}^{3}\right)$ & 9.687 & 3.134 & 6.410 \\
\hline Concentração Corrigida a $11 \% \mathrm{O}_{2}\left(\mathrm{mg} / \mathrm{Nm}^{3}\right)$ & 7.452 & 2.410 & 4.931 \\
\hline Concentração nas Condições da Chaminé $\left(\mathrm{mg} / \mathrm{m}^{3}\right)$ & 3.431 & 1.137 & 2.284 \\
\hline Taxa de Emissão (Kg/h) & 21,63 & 7,15 & 14,39 \\
\hline Vazão Gases Condições Normais Base Seca $\left(\mathrm{Nm}^{3} / \mathrm{h}\right)$ & 3.190 & 3.189 & 3.190 \\
\hline Vazão Gases nas Condições da Chaminé $\left(\mathrm{m}^{3} / \mathrm{h}\right)$ & 6.304 & 6.289 & 6.297 \\
\hline Temperatura dos Gases $\left({ }^{\circ} \mathrm{C}\right)$ & 176 & 174 & 175 \\
\hline Isocinéticas Média (\%) & 100 & 97,5 & 98,8 \\
\hline Velocidade Média dos Gases (m/s) & 26,5 & 26,5 & 26,5 \\
\hline Umidade dos Gases (\%) & 13,2 & 13,4 & 13,3 \\
\hline Teor de Dióxido de Carbono (\%) & 5,1 & 5,3 & 5,2 \\
\hline Teor de Oxigênio (\%) & 11,9 & 11,7 & 11,8 \\
\hline Teor de Nitrogênio (\%) & 83 & 83 & 83 \\
\hline
\end{tabular}


Tabela 5. Emissão de óxidos de nitrogênio na entrada do filtro de mangas

\begin{tabular}{cccccccc}
\hline Amostragem & $\begin{array}{c}\text { Concentração } \\
\text { Condições } \\
\text { Normais Base }\end{array}$ & $\begin{array}{c}\text { Seca } \\
\left(\mathrm{mg} / \mathrm{Nm}^{3}\right)\end{array}$ & $\begin{array}{c}\text { Concentração } \\
\text { Corrigida } \mathrm{O}_{2} \\
\left(\mathrm{mg} / \mathrm{Nm}^{3}\right)\end{array}$ & $\begin{array}{c}\text { Vazão do Gás } \\
\text { nas } \\
\text { Condições }\end{array}$ & $\begin{array}{c}\text { Taxa de } \\
\text { Emissão } \\
(\mathrm{kg} / \mathrm{h})\end{array}$ \\
\cline { 3 - 5 } 1 & 204,4 & 314,5 & 292,1 & 224,7 & 3.190 & 0,652 \\
\hline 2 & 237,7 & 365,6 & 339,5 & 261,2 & 3.190 & 0,758 \\
\hline 3 & 191,1 & 293,9 & 272,9 & 209,9 & 3.190 & 0,609 \\
\hline 4 & 268,9 & 413,8 & 376 & 289,2 & 3.190 & 0,858 \\
\hline
\end{tabular}

Nas tabelas 6 e 7 encontram-se os dados coletados na saída do equipamento.

Tabela 6. Emissão de material particulado na saída do filtro de mangas

\begin{tabular}{|c|c|c|c|}
\hline Parâmetro & $\begin{array}{c}\text { Amostragem } \\
1\end{array}$ & $\begin{array}{c}\text { Amostragem } \\
2\end{array}$ & Média \\
\hline Concentração Condições Normais Base Seca $\left(\mathrm{mg} / \mathrm{Nm}^{3}\right)$ & 21,94 & 10,98 & 16,46 \\
\hline Concentração Corrigida a $7 \% \mathrm{O}_{2}\left(\mathrm{mg} / \mathrm{Nm}^{3}\right)$ & 46,54 & 26,50 & 36,52 \\
\hline Concentração Corrigida a $8 \% \mathrm{O}_{2}\left(\mathrm{mg} / \mathrm{Nm}^{3}\right)$ & 43,22 & 24,61 & 33,91 \\
\hline Concentração Corrigida a $11 \% \mathrm{O}_{2}\left(\mathrm{mg} / \mathrm{Nm}^{3}\right)$ & 33,24 & 18,93 & 26,09 \\
\hline Concentração nas Condições da Chaminé $\left(\mathrm{mg} / \mathrm{m}^{3}\right)$ & 14,10 & 7,20 & 10,65 \\
\hline Taxa de Emissão (Kg/h) & 0,077 & 0,039 & 0,058 \\
\hline Vazão Gases Condições Normais Base Seca $\left(\mathrm{Nm}^{3} / \mathrm{h}\right)$ & 3.519 & 3.555 & 3.537 \\
\hline Vazão Gases nas Condições da Chaminé $\left(\mathrm{m}^{3} / \mathrm{h}\right)$ & 5.471 & 5.448 & 5.460 \\
\hline Temperatura dos Gases $\left({ }^{\circ} \mathrm{C}\right)$ & 101 & 102 & 101 \\
\hline Isocinéticas Média (\%) & 98,6 & 98,9 & 98,7 \\
\hline Velocidade Média dos Gases (m/s) & 16,74 & 16,67 & 16,71 \\
\hline Umidade dos Gases (\%) & 6,54 & 4,89 & 5,72 \\
\hline Teor de Dióxido de Carbono (\%) & 3,20 & 2,7 & 2,95 \\
\hline Teor de Oxigênio (\%) & 14,4 & 15,2 & 14,8 \\
\hline Teor de Nitrogênio (\%) & 82,4 & 82,1 & 82,3 \\
\hline
\end{tabular}


Tabela 7. Emissão de óxidos de nitrogênio na saída do filtro de mangas

\begin{tabular}{ccccccc}
\hline Amostragem & $\begin{array}{c}\text { Concentração } \\
\text { Condições } \\
\text { Normais Base } \\
\text { Seca }\end{array}$ & $\begin{array}{c}\text { Concentração } \\
\text { Corrigida } \mathrm{O}_{2} \\
\left(\mathrm{mg} / \mathrm{Nm}^{3}\right)\end{array}$ & $\begin{array}{c}\text { Vazão do Gás } \\
\text { nas } \\
\text { Condições }\end{array}$ & $\begin{array}{c}\text { Taxa de } \\
\text { Emissão } \\
(\mathrm{kg} / \mathrm{h})\end{array}$ \\
\cline { 3 - 5 } & $7 \%$ & $8 \%$ & $11 \%$ & $\begin{array}{c}\text { Base Seca } \\
\left(\mathrm{Nm}^{3} / \mathrm{h}\right)\end{array}$ & \\
1 & 86,8 & 184,1 & 171 & 131,5 & 3.519 & 0,305 \\
\hline 3 & 123,8 & 262,5 & 243,8 & 187,5 & 3.519 & 0,436 \\
\hline 4 & 102,9 & 218,3 & 202,8 & 156 & 3.519 & 0,362 \\
\hline & 107,3 & 227,6 & 240,5 & 185 & 3.519 & 0,378 \\
\hline
\end{tabular}

Para todas as considerações das amostragens nas tabelas 4, 5, 6 e 7 os valores calculados são baseados em condições normais na base seca, os quais por não sofrerem correções fundamentadas em altitude local, densidade dos gases e porcentagem de $\mathrm{O}_{2}$ presente devido ao excesso injetado para combustão dos resíduos na caldeira, proporcionam maior precisão no cálculo da eficiência do filtro, sendo o cálculo da taxa de emissão $(\mathrm{kg} / \mathrm{h})$ orientado por esses valores. Observa-se também nos dados apresentados que com a queda da temperatura ocorre uma correspondente queda da porcentagem de umidade presente nos gases com a condensação de parte do vapor d' água, o que demonstra situação já citada neste trabalho sobre a importância do isolamento térmico, de forma que o ponto de orvalho não seja atingido durante o circuito, e principalmente no filtro de mangas. Esta situação traria como consequência a presença de água no estado líquido no tecido das mangas em quantidades acima do suportado, saturando o elemento filtrante e dificultando a passagem dos gases, resultando finalmente em uma maior queda de pressão no equipamento.

Com base nos números encontrados de emissão tanto para material particulado quanto para óxidos de nitrogênio é possível estimar os valores de eficiência alcançados pelo filtro de mangas durante a operação. Nas tabelas 8 e 9 são apresentados os resultados obtidos. 
Tabela 8. Eficiência filtro de mangas para material particulado

\begin{tabular}{lccc}
\hline Parâmetro & Amostragem 1 & Amostragem 2 & Média \\
\hline Taxa de Emissão (kg/h) - Entrada Filtro & 21,63 & 7,15 & 14,39 \\
Taxa de Emissão (kg/h) - Saída Filtro & 0,077 & 0,039 & 0,058 \\
Eficiência Filtro de Mangas (\%) & $\mathbf{9 9 , 6 4}$ & $\mathbf{9 9 , 4 5}$ & $\mathbf{9 9 , 5 5}$ \\
\hline
\end{tabular}

Tabela 9. Eficiência filtro de mangas para óxidos de nitrogênio

\begin{tabular}{lccccc}
\hline Parâmetro & $\begin{array}{c}\text { Amostragem } \\
\mathbf{1}\end{array}$ & $\begin{array}{c}\text { Amostragem } \\
\mathbf{2}\end{array}$ & $\begin{array}{c}\text { Amostragem } \\
\mathbf{3}\end{array}$ & $\begin{array}{c}\text { Amostragem } \\
\mathbf{4}\end{array}$ & Média \\
& 0,652 & 0,758 & 0,609 & 0,858 & 0,734 \\
$\begin{array}{l}\text { Taxa de Emissão (kg/h) - } \\
\text { Entrada Filtro }\end{array}$ & & & & & \\
$\begin{array}{l}\text { Taxa de Emissão (kg/h) - Saída } \\
\text { Filtro }\end{array}$ & 0,305 & 0,436 & 0,362 & 0,378 & 0,370 \\
\begin{tabular}{l} 
Eficiência Filtro de Mangas (\%) \\
\hline
\end{tabular} & $\mathbf{5 3 , 1 6}$ & $\mathbf{4 2 , 5 6}$ & $\mathbf{4 0 , 5 7}$ & $\mathbf{5 6 , 0 0}$ & $\mathbf{4 8 , 2 8}$ \\
\hline
\end{tabular}

A tabela 8 mostra claramente que o filtro de mangas Dinaflux $8 \times 7-30$, objeto de estudo deste trabalho, alcançou a eficiência típica para este tipo de coletor, demonstrando que os parâmetros de dimensionamento utilizados foram adequados, resultando entre outros fatores na formação da camada externa de pó na manga (cake) suficiente para que se atingisse a eficiência almejada com a menor queda de pressão possível. Em outra frente pode-se observar na tabela 9 que a eficiência da filtragem para óxidos de nitrogênio é muito menor, porém os requisitos de emissões para este componente também são muito menores.

Atualmente a resolução CONAMA n 382 de 26/12/2006 estabelece como limite de emissão para material particulado o valor de $200 \mathrm{mg} / \mathrm{Nm}^{3}$ e como limite de emissão para 
óxidos de nitrogênio o valor de $350 \mathrm{mg} / \mathrm{Nm}^{3}$, valores estes que não foram ultrapassados como pode ser visualizado nas tabelas 6 e 7 .

De forma a fornecer base de comparação entre as especificações técnicas do tecido e suas condições posteriores ao uso, a análise do elemento filtrante foi realizada em dois estados, sendo o primeiro com o tecido nas condições anteriores ao ciclo de limpeza do sistema jato pulsante e o segundo com o tecido nas condições após a atuação do sistema jato pulsante. $O$ resultado das análises dos elementos filtrantes encontra-se nas tabelas 10 e 11 a seguir.

Tabela 10. Resultados das análises dos elementos filtrantes (parte 1)

\begin{tabular}{|c|c|c|c|c|c|}
\hline \multicolumn{6}{|c|}{ Ensaios e Unidades de Medidas } \\
\hline \multirow[t]{2}{*}{$\begin{array}{l}\text { Especificação } \\
\text { Técnica }\end{array}$} & $\begin{array}{c}\text { Gramatura } \\
\left(\mathrm{g} / \mathrm{m}^{2}\right)\end{array}$ & $\begin{array}{c}\text { Espessura } \\
(\mathrm{mm})\end{array}$ & $\begin{array}{c}\text { Permeabilidade } \\
\text { ( } 1 / \mathrm{min}^{\mathrm{m}} \mathrm{dm}^{2} \text { à } 20 \\
\mathrm{mmCA})\end{array}$ & $\begin{array}{l}\text { Resistência à } \\
\text { Tração } \\
\text { Longitudinal } \\
\text { (daN) }\end{array}$ & $\begin{array}{c}\text { Resistência à } \\
\text { Tração } \\
\text { Transversal } \\
\text { (daN) }\end{array}$ \\
\hline & 750 & 0,8 & 37 & 155 & 155 \\
\hline \multicolumn{6}{|c|}{ Resultados dos Ensaios } \\
\hline Manga - Suja & 787 & 1,05 & 17,2 & 123 & 167 \\
\hline Manga - Limpa & 780 & 1,02 & 20,3 & 123 & 167 \\
\hline
\end{tabular}

Tabela 11. Resultados das análises dos elementos filtrantes (parte 2)

\begin{tabular}{lcc}
\hline \multicolumn{3}{c}{ Ensaios e Unidades de Medidas } \\
\hline $\begin{array}{l}\text { Especificação } \\
\text { Técnica }\end{array}$ & $\begin{array}{c}\text { Alongamento } \\
\text { Longitudinal (\%) }\end{array}$ & $\begin{array}{c}\text { Alongamento Transversal } \\
\text { (\%) }\end{array}$ \\
& 10 & 10 \\
Manga - Suja & 12 & 17 \\
Manga - Limpa & 12 & 17 \\
\hline
\end{tabular}


Através dos dados das tabelas 10 e 11 é possível atestar a formação da camada externa de pó (cake) com espessuras entre 0,25 mm e 0,22 mm, que Saleem, M.; Krammera, G. (2007), mostra estar dentro dos padrões normais para filtros de mangas. Verifica-se nos dados valores coerentes de gramatura em decorrência da presença da membrana expandida de teflon (PTFE), que promove um menor acúmulo de particulado depositado sobre a superfície filtrante da amostra analisada devido à facilidade de desprendimento do pó. Ao analisar-se a correspondência entre os resultados de gramatura, espessura e permeabilidade, é possível visualizar valores maiores de gramatura e espessura para as condições de manga suja com menor permeabilidade, e valores menores de gramatura e espessura com decorrente aumento da permeabilidade do tecido mostrando claramente a atuação do sistema de limpeza. Os dados vão de encontro ao usual para aplicações com coletores tipo filtro de mangas, e são avalizados pelo teste de permeabilidade que demonstrou resultados dentro do esperado para meios filtrantes com membrana, atestando assim o uso de velocidade de filtragem coerente a aplicação. Os dados de resistência à tração e alongamento apontam boa resistência mecânica do tecido demonstrando a não ocorrência de modificações de ordem química e estrutural neste, situação esperada em um sistema que apresente o correto funcionamento. Outro ponto que pode ser notado são os valores maiores de alongamento transversal e resistência à tração transversal, atingidos após o uso do elemento filtrante no experimento, o que evidencia a fadiga do material causada pelo sistema de limpeza jato pulsante, atuante com vetor na direção transversal. A análise visual, onde se confirmou a não presença de danos estruturais no tecido e particulado na face interna do elemento filtrante, em conjunto a todas as demais análises do elemento filtrante corroboram os resultados e dados obtidos a respeito de eficiência do equipamento e seu correto funcionamento.

\section{CONCLUSÃO}

A partir dos resultados obtidos e avaliados, o Sistema de Filtragem a Seco mostrou-se viável, apresentando alta eficiência de coleta com baixa pressão diferencial. É 
ISSN 1980-0827
Volume 9, Número 11, 2013

Saúde, Saneamento e

Meio Ambiente

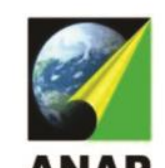

ANAP

evidente que a regulagem da caldeira influencia diretamente no equipamento de tratamento de gases, porém em condições normais de regulagem e funcionamento desta, o sistema mostra-se funcional. Para aplicação em escala industrial, recomenda-se a adoção de parâmetros de dimensionamento e de proteção que previnam falhas na descarga de material acrescido de sistema de combate a incêndio. O Sistema de Filtragem a Seco, assim projetado, irá apresentar altas eficiências de filtragem sem a geração de efluente líquido para tratamento ou disposição posterior.

\section{AGRADECIMENTOS}

Este projeto de pesquisa tem como principal colaborador a TERSEL Equipamentos Industriais Itda, fabricante de todos os equipamentos componentes do sistema de tratamento de gases e detentora do conhecimento aplicado no estudo incluindo os dados resultantes das coletas de emissões de gases, a qual os autores agradecem. Agradecemos também a Açúcar Guarani - Unidade de Olímpia a qual permitiu a instalação do equipamento em uma de suas caldeiras, e a JAPH- Serviços Analíticos, empresa contratada para realizar as coletas e análises das emissões. A todos muito obrigado.

\section{REFERÊNCIAS}

American Conference of Governmental Industrial Hygienists - Committee on Industrial Ventilation, 2001, "Industrial Ventilation: a manual of recommended practice", $24^{\mathrm{a}}$ Edição, Cincinnati, Ohio.

Conselho Nacional do Meio Ambiente. Resoluções do Conama: resoluções vigentes publicadas entre julho de 1984 e novembro de 2008 - 2. ed. / Conselho Nacional do Meio Ambiente. - Brasília: Conama, 2008.

L"offler, F.; Dietrich, H.W. (1988), Flatt, Dust Collection with Bag Filters and Envelope

Filters, Friedr. Vieweg \& Sons, Braunschweig/Wiesbaden, Germany. 
Manual de Normas Técnicas CETESB, Normas L9.210 - Análise dos Gases de Combustão Através do Aparelho Orsat, L9.221 - Dutos e Chaminés de Fontes Estacionárias - Determinação de Pontos de Amostragens, 1990.

Manual de Normas Técnicas CETESB, Normas L9.222 - Dutos e Chaminés de Fontes Estacionárias - Determinação da Velocidade e Vazão dos Gases, L9.223 - Dutos e Chaminés de Fontes Estacionárias - Determinação da Massa Molecular Seca e do Excesso de Ar do Fluxo Gasoso, L9.229 - Dutos e Chaminés de Fontes Estacionárias Determinação de Óxidos de Nitrogênio, 1992.

Manual de Normas Técnicas CETESB, Norma L9.224 - Dutos e Chaminés de Fontes Estacionárias - Determinação da Umidade dos Efluentes, 1993.

Manual de Normas Técnicas CETESB, Norma L9.225 - Dutos e Chaminés de Fontes Estacionárias - Determinação de Material Particulado, 1995.

Mesquita, A.L.S.; Guimarães, F.A.; Nefussi, N, 1977. "Engenharia de Ventilação Industrial", $1^{a}$ Edição, São Paulo, Edgard Blucher Ltda.

Saleem, M.; Krammera, G., 2007. "Effect of filtration velocity and dust concentration on cake formation and filter operation in a pilot scale jet pulsed bag filter", Journal of Hazardous Materials, 144 (2007) 677-681. 